\title{
Junta Consultiva y Comisiones Investigadoras en la Provincia de Buenos Aires: usos de la escala para pensar el conflicto peronismo-antiperonismo
}

\author{
Advisory Board and Investigative Commissions in the Buenos Aires province: \\ uses of scale to think peronismo- antiperonism conflict.
}

\author{
Silvana Gabriela Ferreyra \\ Centro de Estudios Históricos (Universidad Nacional de Mar del Plata) \\ Consejo Nacional de Investigaciones Científicas y Técnicas, Argentina
}

\begin{abstract}
Resumen
Este artículo intenta reflexionar en la intersección entre dos tópicos: las historias provinciales/locales y los estudios sobre antiperonismo en la "libertadora". El punto de partida es el análisis de la Junta Consultiva y las Comisiones Investigadoras en la Provincia de Buenos Aires. El objetivo principal es apreciar en distintas dimensiones la radicalidad del conflicto peronismo-antiperonismo durante la "revolución libertadora." Por un lado, identificamos similitudes y diferencias en las alianzas políticas de los distintos niveles administrativos bonaerenses con respecto al gobierno nacional. Nos encontramos con un escenario complejo y heterogéneo que evidencia tramas alternativas al binomio peronismoantiperonismo. Por otro lado, analizamos los expedientes de las comisiones investigadoras para estudiar la recepción de las políticas "desperonizadoras" entre la "gente ordinaria". En esta línea, proponemos una mirada microanalítica que resalta la intensidad del conflicto. Las denuncias de las comisiones muestran cruces entre lo personal y lo político y evidencian la tensión social que una década de peronismo había instalado a "ras de suelo". En definitiva, el artículo pretende constituirse en un pequeño laboratorio de trabajo para testear usos diversos de la escala en los estudios provinciales.
\end{abstract}

Palabras claves

Escala, microhistoria, historia provincial, antiperonismo, "revolución libertadora"

\begin{abstract}
This paper aims to think about the intersection between two subjects: provincial/local histories and studies about Antiperonism at "Libertadora". The starting point is given in the analysis of Advisory Board and Investigative Commissions in the Buenos Aires province. The main objective means to appreciate the radicality of Peronism-Antiperonism conflict for the period of "liberating revolution" in several dimensions. On one hand, we identify both similarities and differences in distinct levels of bonaerense administration in relation to national government. We find a complex and heterogeneous scenario which shows alternative positions to Peronism-Antiperonism pairing. On the other hand, we analyse files of Investigative Commissions in order to study the reception of "desperonización" policies among "common people". In this way, we propose a microanalytical point of view which highlights the intensity of this conflict. Denunciations of Commissions exhibit crossings
\end{abstract}




\section{Silvana Gabriela Ferreyra}

between personal and political matters and demonstrate social tension "au ras du sol" installed after one decade of Peronism. In short, the paper expects to became a little laboratory for testing several uses of scale in provincial studies.

\section{Keywords}

Scale, microhistory, provincial history, Antiperonism, "liberating revolution"

\section{Introducción}

Las dimensiones local y provincial lograron un importante espacio en el diálogo historiográfico de las últimas décadas. Dos motivaciones distintas, aunque no excluyentes, parecen haber jugado algún papel a la hora de elegir un recorte subnacional para analizar problemas históricos. Una de ellas, menos confesa pero no por eso menos efectiva, se apoya en los requerimientos de la profesionalización del historiador. Las exigencias para encontrar una "vacancia" en un campo que crece a un ritmo vertiginoso, podían resolverse seriamente recurriendo al análisis de distintos interrogantes en espacios más o menos restringidos (regiones, provincias, ciudades). La otra, enunciada como elección metodológica, resulta de suponer que en estos niveles de análisis pueden situarse los procesos causales de los cuales dependen los procesos históricos que se colocan bajo la lupa.

A diferencia de lo que ha ocurrido con los estudios sobre otros períodos de la historia (en particular nos referimos al período peronista), el análisis regional de los sucesos políticos acontecidos durante la "revolución libertadora" se encuentra todavía en desarrollo. ${ }^{1}$ En esta encrucijada también el trabajo de César Tcach se constituyó como referencia, ${ }^{2}$ analizando las estrategias políticas de las distintas fuerzas y las nuevas correlaciones que se tejieron con la proscripción del peronismo para el escenario cordobés. El modo en que las tramas no siempre se correlacionaban con las que se tejían para la escena nacional, permitía advertir un país federal, con pactos territoriales diferenciados y un panorama nacional complejo. ${ }^{3}$ Pero la hipótesis de Tcach iba más allá de advertir sobre esta diversidad

\footnotetext{
${ }^{1}$ El análisis del gobierno nacional de la "revolución libertadora" ha sido estudiado minuciosamente por María Estela Spinelli. Los vencedores vencidos. El antiperonismo y la "revolución libertadora." Buenos Aires, Biblos, 2005 y por María Sáenz Quesada. La Libertadora. De Perón a Frondizi. 19551958. Historia pública y secreta. Buenos Aires, Sudamericana, 2007.

2 César Tcach. "Partidos y pactos políticos en la Córdoba Libertadora (1955-1958)". Revista Estudios, № 3. Córdoba, 1994, pp. 17-30. Para el período peronista véase Darío Macor y César Tcach, (editores). La invención del peronismo en el interior del país. Santa Fe, Ediciones UNL, 2003 y Darío Macor y César Tcach, (editores.). La invención del peronismo en el interior del país II. Santa Fe, Ediciones UNL, 2013.

${ }^{3}$ Este ejercicio, movidos por motivaciones varias, se repitió para otros escenarios provinciales. Véase para el caso de Río Negro el capítulo de Graciela Iuorno. "La provincialización de Río Negro. Interregno y conflictos de intereses nacionales y locales", en Graciela Iuorno y Edda Crespo, (coordinadoras), Nuevos Espacios. Nuevos problemas. Los territorios nacionales. Neuquén, EducoUniversidad Nacional del Comahue-Universidad Nacional de la Patagonia San Juan Bosco-Cehepyc Editores, 2008.; para Santa Cruz el texto de Aixa Bona y Juan Vilaboa. "La transición política de los territorios nacionales y la caída del peronismo: el caso de Santa Cruz". Estudios, N2 22. Córdoba, 2009, pp. 49-60; para Tucumán la ponencia de Leandro Lichtmajer y Lucía Santos Lepera. "Repensando el
} 


\section{Junta Consultiva y Comisiones Investigadoras en la Provincia de Buenos Aires}

de casos; su trabajo buscaba demostrar que una mirada situada en las rencillas políticas dejaba en evidencia el escaso peso que el conflicto peronismoantiperonismo tenía en la agenda cotidiana del mundo posperonista.

Motivados por este problema, en este artículo decidimos analizar dos objetos que ya hemos indagado en distintos momentos de nuestra trayectoria de investigación. Se trata de dos observables clásicos para un enfoque institucional: las juntas consultivas y las comisiones investigadoras, ambas creadas por el gobierno de facto de la autodenominada "revolución libertadora". En cuanto al primer caso, su tratamiento se presentó de modo tangencial en mi proyecto doctoral. ${ }^{4} \mathrm{Al}$ interesarme por el itinerario del Partido Socialista Democrático en la Provincia de Buenos Aires, estudié su participación en la Junta Consultiva Nacional y Provincial para reflexionar sobre los enfrentamientos y acuerdos con las restantes fuerzas políticas. En este análisis aparecieron claves alternativas a las fijadas por el tradicional binomio, tal como había señalado Tcach en su investigación. Años más tarde, como un modo de profundizar mis conocimientos sobre el antiperonismo más radicalizado, me interesé por indagar en la experiencia de las comisiones investigadoras, abordando nuevamente el conflicto peronismo-antiperonismo, pero desde otras lecturas y enfoques. ${ }^{5}$

Si bien se trata de entradas similares, rescataremos en este artículo los tratamientos diferenciales a los que fueron sometidos en nuestra investigación, atendiendo a diferentes usos de la escala para el análisis del período 1955-1958. En esta línea, buscaremos pensar el conflicto peronismo-antiperonismo teniendo en cuenta diferentes maneras de registrar lo micro. ${ }^{6}$ En el primer aparatado, nos concentraremos en las juntas consultivas desde la lógica modelo/desviaciones, ${ }^{7}$ usual en las historias locales que viene construyendo la historiografía. Desde esta óptica, podemos referir al modo en que los casos regionales y provinciales muestran articulaciones políticas que se suponen como reflejos o distorsiones frente a lo

\footnotetext{
golpe de 1955 desde una perspectiva provincial: Iglesia católica y radicalismo en Tucumán”, ponencia presentada en Tercer Congreso de Estudios sobre el Peronismo, San Salvador de Jujuy, Jujuy, 2012.

4 Silvana Ferreyra. Socialismo y antiperonismo: el Partido Socialista Democrático. Transformación partidaria y dinámica política en tiempos de proscripción (Provincia de Buenos Aires, 1955-1966). Tesis de Doctorado. Mar del Plata, UNMdP, 2012.

${ }^{5}$ Mi proyecto actual como investigadora en CONICET se denomina Prácticas e imaginación política antiperonistas: las comisiones investigadoras durante la "revolución libertadora".

6 Para seguir algunas discusiones teóricas sobre el tema véase Jacques Revel. "Microanálisis y construcción de lo social". Anuario del IEHS, № 10. Tandil, 1995, pp. 125-143; Justo Serna y Anaclet Pons. "Más cerca, más denso. La historia local y sus metáforas", en Sandra Fernández, (compiladora), Más allá del territorio. La historia regional y local como problema. Discusiones, balances y proyecciones. Rosario, Prohistoria, 2007 y Bernard Lepetit. "Sobre a escala na história”, en Jacques Revel, (editor), Jogos de escala: a experiencia da micro-analis. Rio de Janeiro, Fundación Getulio Vargas, 1998.

7 Nos permitimos aquí una extrapolación desde el campo de la historia intelectual, propuesta por Elias Palti. El autor considera que este esquema fue el propio de la historia de las ideas latinoamericanas, en la medida en que esta disciplina se centró en la definición de tipos "puros" o modelos originados en Europa y la identificación de las "desviaciones" ocurridas una vez que estas ideas eran trasladadas a un medio supuestamente extraño y hostil a las mismas. Véase Elías Palti. "De la historia de 'ideas' a la historia de los 'lenguajes políticos'. Las escuelas recientes de análisis conceptual. El panorama latinoamericano”. Anales Nueva Época, № 7/8. 2004, p. 70.
} 


\section{Silvana Gabriela Ferreyra}

ocurrido en un espacio capitalino que se modeliza. En el segundo apartado nos concentramos en las comisiones investigadoras, apostando a colocar el foco en el análisis de actores históricos en contextos múltiples y pensando lo micro como dimensión de análisis.

Nos concentraremos en la Provincia de Buenos Aires como experiencia de análisis, elección fácilmente justificable al tratarse de la provincia con mayor peso económico y demográfico de la Argentina. No obstante, no podemos dejar de aclarar que nuestro objetivo principal es advertir las variaciones de un enfoque situado antes que conocer minuciosamente lo que ha ocurrido en la provincia de Buenos Aires durante estos años. ${ }^{8}$

\section{Juntas Consultivas}

La Junta Consultiva Nacional fue creada el 28 de octubre de 1955, bajo la presidencia de facto de Eduardo Lonardi, aunque recién tomó cierto dinamismo a partir de la asunción de Pedro Eugenio Aramburu, pocos días después. Fue presidida por el vicepresidente, almirante Isaac Francisco Rojas, e integrada por miembros de los distintos partidos políticos "democráticos" (fueron excluidos peronistas y comunistas). Sin atender a representaciones mayoritarias o proporcionales, estos partidos políticos fueron convocados por el gobierno militar en igualdad de número, aportando cuatro miembros cada uno: la Unión Cívica Radical, el Partido Demócrata Nacional, el Partido Socialista, el Partido Demócrata Cristiano, el Partido Demócrata Progresista y Unión Federal. El discurso democrático y republicano que paradojalmente- representaba al golpe de 1955 buscaba homologar esta institución con el poder legislativo, funcionando en el propio Congreso Nacional. Sin embargo, se trataba de un organismo únicamente asesor, que trabajaba a partir de las sugerencias enviadas por el Ejecutivo, sin elevar proyectos propios. Su accionar se puede seguir minuciosamente a través de los diarios de sesiones, aunque algunas reuniones no se reprodujeron por definirse como secretas. ${ }^{9}$ Con estas cualidades, las juntas consultivas se reprodujeron en todos los niveles, tanto provinciales como municipales, lo que habilita una lectura escalar de su funcionamiento. En esta línea, rastreamos algunos ejes que nos permitan identificar similitudes y diferencias entre la Junta Nacional y sus homologas regionales.

\footnotetext{
${ }^{8}$ El registro empírico de lo ocurrido en la provincia de Buenos Aires, al menos desde la perspectiva de la historia política institucional, puede seguirse -aunque exiguamente- en manuales sobre la historia de la provincia. Véase Ricardo Levene. Historia de la Provincia de Buenos Aires y formación de sus pueblos. La Plata, Taller de Impresiones Oficiales, 1941 y Osvaldo Barrenche (director). Historia de la Provincia de Buenos Aires. Tomo 5: Del primer peronismo a la crisis de 2001. Buenos Aires, UNIPEEdhasa, 2014.

${ }^{9}$ Para el caso de los diarios de sesiones parlamentarios también se ha señalado que sólo se registran los debates en las sesiones públicas -y no las reuniones de comisión ni de los bloques partidarios-, ocultando buena parte de las discusiones de fondo y proporcionando una imagen sesgada a favor de la armonía partidaria. Al respecto, véase Marcela Ferrari y Mariana Pozzoni. "Tensiones y conflictos en el peronismo: un análisis a través de la Legislatura bonaerense, 1973-1976“. Cahiers des Amériques latines, $\mathrm{N}^{\circ}$ 75. París, 2014, pp. 147-176.
} 


\section{Junta Consultiva y Comisiones Investigadoras en la Provincia de Buenos Aires}

Como primer eje podemos analizar el modo en que las disidencias de distintas fuerzas políticas hacia el gobierno nacional implicaron sucesivos alejamientos de la institución. En la medida en que estos movimientos no ocurrieron de forma homogénea en los distintos territorios, abrir el "juego de escalas" nos brinda la posibilidad para pensar la especificidad de las alianzas en cada región. De hecho, el retraso que ocasionó la propia dinámica burocrática para disponer la normativa necesaria a efectos de reproducir en cada provincia un organismo similar al nacional, fue el primer diferencial en las condiciones políticas.

En Buenos Aires la constitución de la Junta llegó recién dos meses después que la nacional: el 3 de enero de 1956. Ese primer desfasaje implicó que ya en su constitución inicial estuvieran ausentes los nacionalistas. De cualquier forma, la brecha entre el interventor Coronel Emilio Augusto Bonecarrere y el coronel Arturo Ossorio Arana parece haber sido menor que la que la bibliografía marca entre Lonardi y Aramburu. Los cambios en el gabinete provincial se limitaron a las carteras de Hacienda y de Gobierno, probablemente porque Ossorio Arana no era fiel representante del sector nacionalista del Ejército, sino simplemente un oficial no demasiado liberal. ${ }^{10}$ La conformación inicial de la Junta estuvo dada por Velma Celin Obieta de Rodríguez y José Clusellas (Partido Demócrata Progresista); Emilio J. Hardoy y Pablo González Bergez (conservadores); Juan Prat y Doroteo Ireneo Drake (Unión Cívica Radical); José Amado y Mario Seijo (Partido Demócrata Cristiano) y Teodoro Bronzini y Eduardo Schaposnik (PS). Si bien la Junta terminó sus funciones el 3 de marzo de 1958, ${ }^{11}$ su actividad más agitada parece haberse desarrollado durante el año 1956, momento hasta el que tenemos registro de su accionar en el diario de sesiones, cuando se abrió el receso de verano que tuvo cierta continuidad con la detención de actividades para las elecciones de constituyente en julio de 1957 y la posterior paralización por la labor de la convención. Los representantes conservadores dejaron de participar de la misma a fines de julio de 1956, aunque el Partido Demócrata nombró otros en 1957, designando a Julio Rodolfo César y Roberto Sahores. La democracia cristiana, cuyas disidencias con la mayoría de la junta se habían manifestado respecto al debate sobre educación religiosa y en relación a la creación del Departamento Provincial del Trabajo, se retiró definitivamente de la Junta Provincial a fines de septiembre de 1956. Otra particularidad fue la designación de dos integrantes radicales que se vincularían posteriormente a la Unión Cívica Radical del Pueblo, razón por la cual no manifestaron el tipo de disidencia que Oscar López Serrot y Oscar Allende vinculados a la Intransigencia- se encargaban de llevar a la Junta Nacional. ${ }^{12} \mathrm{Al}$

\footnotetext{
10 Alain Rouquié. Poder militar y sociedad política en la Argentina,1943-1973, Vol. II. Buenos Aires, Emecé, p.139.

11 Decreto Provincial № 2646/58

12 En la Junta Consultiva Nacional estuvieron representadas las tres líneas internas del radicalismo: Miguel Ángel Zavala Ortiz por el unionismo, Octavio Gauna por la intransigencia nacional y Oscar López Serrot y Oscar Alende por el Movimiento de Intransigencia y Renovación, ambos leales a Frondizi. Para estos últimos, el único objetivo de la "libertadora" era derrocar a Perón, razón por la cual debía convocar a elecciones inmediatamente. Ello explicaría su posición crítica al gobierno de
} 


\section{Silvana Gabriela Ferreyra}

respecto, Schaposnik (representante del PS) señaló que "la Junta Consultiva de la provincia de Buenos Aires está dando un ejemplo de convivencia política que no se ha podido lograr en el resto del país".13

Asimismo, el enfoque puede extenderse al plano local, introduciendo en la comparativa a las que se denominaron como Comisiones Asesoras Municipales. Estos organismos, a diferencia de las Juntas Consultivas Provinciales y Nacionales, tuvieron inicialmente a una representación corporativa. Es decir, mientras que la composición de las Juntas Consultivas Nacionales y Provinciales contemplaba la representación igualitaria de los partidos "democráticos", las Comisiones Asesoras municipales estuvieron compuestas por "vecinos" distinguidos, representantes de entidades que agrupaban a comerciantes, industriales, obreros y profesionales. Este escenario se modificó tras la aprobación de las "Instrucciones para los Comisionados" en febrero de 1956 y la consideración del municipio como un espacio político, equivalente al de la provincia y la nación. ${ }^{14}$ En la sesión de la Junta Consultiva Provincial donde se discutieron estas instrucciones, Schaposnik denunciaba que en Lomas de Zamora y Pergamino el Partido Socialista le había retirado su apoyo a la Comisión Asesora por la forma discrecional en que el comisionado había procedido para la designación de los miembros. Asimismo, denunciaba la sobrerrepresentación del radicalismo en las comisiones de varias comunas, como General Arenales, Juárez y General Rodríguez. ${ }^{15}$

En efecto, en cada municipio las alianzas políticas se desplegaron de modos particulares, atendiendo a las hegemonías locales y los factores de poder dispuestos en el territorio. Así, en Mar del Plata, la combinación entre la cercanía con la base naval, el arraigo histórico del socialismo local y el alineamiento inicial de un importante sector del movimiento obrero con los "gremios democráticos", convirtió a la ciudad en un coto de poder para los grupos más radicalizados del antiperonismo. El comisionado designado, Celso Aldao, era un médico de la marina simpatizante del radicalismo que se mantuvo en su cargo entre octubre de 1955 y mayo de 1958. La Comisión Asesora jugó un rol activo en casi todos los proyectos que se llevaron adelante. Los socialistas estuvieron mejor posicionados territorialmente que los otros partidos antiperonistas y fueron otras las fuerzas que atravesaron situaciones

\footnotetext{
Aramburu en el seno de la Junta, mientras que los unionistas y la intransigencia nacional colaboraron estrechamente con el gobierno de facto. Los "moderados" de la intransigencia, liderados por Ricardo Balbín, compartían esta posición que a partir de noviembre de 1956 los unirá en la conformación de la Unión Cívica Radical del Pueblo. Aunque no tuvieron representación en la junta consultiva nacional, los balbinistas sí la obtuvieron en la junta bonaerense, a través de Juan Prat. Para más detalles sobre la participación de las distintas fracciones del radicalismo en la "libertadora" véase María Estela Spinelli. Los vencedores..., Op. Cit., pp. 194-196.

${ }^{13}$ Honorable Junta Consultiva de la Provincia de Buenos Aires, Diario de Sesiones, La Plata, Dirección de Impresiones Oficiales. Sesión 18/12/56.

${ }^{14}$ La asociación entre el espacio local y la idea de vecino frente al espacio nacional y la idea de ciudadano ha sido ampliamente debatida en la historiografía. Dos referencias importantes pueden encontrarse en Marcela Ternavasio. Municipio y política, un vínculo conflictivo. Tesis de Maestría. Buenos Aires, FLACSO, 1991 y Luciano De Privitellio. Vecinos y ciudadanos. Buenos Aires, Siglo XXI, 2003.

${ }^{15}$ Honorable Junta Consultiva de la Provincia de... op. cit. Sesión 21/2/56.
} 


\section{Junta Consultiva y Comisiones Investigadoras en la Provincia de Buenos Aires}

"enojosas" y se distanciaron del gobierno local. En primer término, se retiraron los demócratas cristianos, quienes también lo habían hecho de la Junta Provincial. Más tarde, en octubre de 1956, a raíz de desavenencias internas en el partido sobre las posiciones que habían adoptado los representantes en la comisión, los consejeros radicales dejaron de asistir. En octubre de 1957, después del receso abierto por las elecciones de constituyentes, fueron los conservadores quienes no se reintegraron a la junta, quedando en la comisión únicamente socialistas y demócrata progresistas, quienes por otra parte solían avalar las listas del PS a nivel local. Ante esta situación, los representes socialistas (Luis Nuncio Fabrizio y Jorge Lombardo) optaron por renunciar para facilitar la reorganización de la comisión, aunque la cercanía del escenario electoral tornaba ya dificultoso su funcionamiento.

Otro eje a considerar es el modo en que las juntas Consultivas Provinciales pueden mostrar cómo gobiernos nacionales y provinciales del mismo color político disputan por los recursos, atendiendo a las lógicas del centralismo y el federalismo. En concreto, la Junta Consultiva de la Provincia de Buenos Aires reclamó que la intervención solicitase al gobierno nacional la recuperación de las fuentes impositivas, tales como la provincialización de los casinos, la jurisdicción sobre zonas balnearias y puertos, así como los fondos retenidos y percibidos por sus beneficios. De hecho, a partir de los reclamos se concretaron algunos avances en torno a la recuperación de los derechos en materia de energía, a la situación del Banco Provincia con relación al régimen bancario nacional, a la participación de la provincia en los impuestos internos unificados y al régimen de los convenios de superposición impositiva.

En resumen, la visión escalar de juntas consultivas nacionales, provinciales y locales parece mostrar -atendiendo a diferentes ejes- articulaciones políticas específicas, regidas por lógicas territoriales propias.

\section{Comisiones Investigadoras}

La "libertadora" mostró ser un golpe de estado con cierta imaginación institucional: junto a las Juntas Consultivas creó las Comisiones Investigadoras, uno de los engranajes principales de la "desperonización". Este proceso se basó inicialmente en la consideración del peronismo como corruptor del sistema político y social, profundizándose al oficializarse su definición como enemigo "totalitario" del sistema democrático y de la nación misma. El mismo fue ganando en extensión y capilaridad, desde las acusaciones a las segundas filas partidarias hasta el intento por eliminar al peronismo como identidad política. Otros de los mecanismos y herramientas que se utilizaron para tal fin fueron la eliminación de las denominaciones de Perón y Eva Perón a distintos espacios públicos; la salida de circulación de los libros de lectura peronistas; la prohibición de cantar la marcha partidaria; la liberación de los presos políticos y militares que habían combatido al peronismo; el recorte de los derechos políticos a Perón y su personal político; la intervención de la CGT; la disolución del Partido Peronista y la confiscación de sus 


\section{Silvana Gabriela Ferreyra}

bienes; la puesta en vigencia de cláusulas discriminatorias para acceder a cargos o concursos; cesantías en reparticiones de salud pública, educación, justicia, fuerzas armadas, etc.

La Comisión Nacional de Investigaciones fue instituida por decreto el 7 de noviembre de 1955 durante la presidencia de Lonardi y terminó su accionar el 31 de marzo de 1956 durante el gobierno de Aramburu. Creada con el objeto de investigar las irregularidades producidas durante la gestión que habían derrocado, junto a su faz represiva dirigida hacia las personas vinculadas de distinto modo al gobierno peronista, las comisiones buscaban deslegitimar un régimen que había sido expulsado del gobierno pese a su amplio apoyo popular. Estuvo conformada por cuatro integrantes civiles y militares, ${ }^{16}$ número que rápidamente consideraron no les permitía cumplir las amplias funciones que se habían atribuido. Un organismo complementario fue la Junta Nacional de Recuperación Patrimonial, facultada para comprobar el origen lícito o ilícito de los bienes de funcionarios públicos y de terceros vinculados a la Administración y recuperarlos para la órbita estatal.

Por su parte, a partir del artículo $4^{\circ}$ de su reglamento interno, la Comisión Nacional quedaba autorizada para nombrar todas las subcomisiones que fuesen necesarias, con el fin de investigar cada rama o dependencia. De este modo se crearon 60 subcomisiones, cuya lógica de apertura respondía tanto a la estructura burocrática (ministerios, secretarías, poderes del estado) como al ritmo y la lógica de la investigación (casos y personajes resonantes). Este número ya importante se multiplicó cuando cada Intervención Provincial o Territoriana creó una comisión para investigar su área de influencia, la que a su vez autorizó sub comisiones que, además de reproducir el esquema anterior de ministerios y secretarías, agregaban una para cada comuna, departamento o municipio, según los nombres que tomaba en cada espacio la administración de lo local. La descentralización administrativa y territorial que se generó terminó en la apertura de más de 400 subcomisiones a lo largo y lo ancho de Argentina.

En particular, la comisión investigadora de la Provincia de Buenos Aires se dividió en 6 comisiones ministeriales, 1 comisión del poder legislativo, 110 comisiones municipales y 7 para el poder judicial (una por cada distrito). Si bien los gobernadores Mercante y Aloe polarizaron la investigación de los hechos más salientes (reuniendo 834 sumarios), debemos considerar al menos otros 4132 sumarios recibidos de los cuales 764 se elevaron a la justicia del crimen. ${ }^{17}$

La relación entre comisiones y poder judicial fue compleja. Las mismas tuvieron amplias atribuciones: realizar indagatorias a sospechosos y testigos, disponer y mantener incomunicaciones y detenciones, allanar domicilios con auxilio de la

\footnotetext{
${ }^{16}$ Los integrantes fueron el Contraalmirante Leonardo McLean (presidente) y los vocales: General de Brigada Luis Rodolfo González, el Brigadier mayor Federico Fernando Antonio Ruiz y el Doctor Rodolfo Medina. Véase Vicepresidencia De La Nación - Comisión Nacional De Investigaciones . Documentación, Autores y Cómplices de las irregularidades cometidas durante la segunda tiranía. Buenos Aires, servicio oficial, 1958.

17 Vicepresidencia De La Nación - Comisión Nacional De Investigaciones. Documentación..., Op. Cit., Tomo V, pp. 5-166
} 


\section{Junta Consultiva y Comisiones Investigadoras en la Provincia de Buenos Aires}

fuerza pública, dictar medidas precautorias para evitar la desaparición de los bienes ilícitamente adquiridos, intervenir libros y contabilidades de entidades, disponer exámenes periciales, extraer las constancias necesarias de registros de escribanos, bancos, Dirección General Impositiva, registro de la propiedad; solicitar el auxilio de la fuerza pública cuando lo considere necesario; entre otras. Aunque sus funciones se superponían en parte con las del poder judicial (algo que no parece extraño en un gobierno de facto), las mismas no incluían la potestad para resolver los casos. En efecto, las comisiones realizaban investigaciones que derivaban, según el delito en cuestión, a la justicia penal o a cada ministerio y repartición para continuar un sumario administrativo. Asimismo, debe señalarse que existía un nivel de incertidumbre importante en torno a los resultados de los procesos, advirtiendo como ya hemos mencionado en el párrafo anterior- que sólo un porcentaje menor de las investigaciones impulsadas por las comisiones fueron derivadas a la justicia. No hay dudas sobre la posibilidad de pensar las comisiones desde la lógica modelo/desviaciones, atendiendo a los mismos o similares problemas que recuperamos en torno a la experiencia de las Juntas Consultivas. Si bien la dimensión partidaria parece menos tangible en este caso, pues las comisiones se formaron con civiles y militares vinculados al área que se buscaba indagar antes que con representantes de fuerzas políticas, la tensión centralismo/ federalismo continúa siendo un eje claro. Al respecto podemos decir que si bien la Comisión Nacional fue concebida de manera centralizada, algunas provincias hicieron llegar sus reclamos de autonomía. Por ejemplo, uno de los procedimientos establecidos indicaba que todas las subcomisiones creadas por las provincias debían elevar sus resultados a la Comisión Provincial y que ésta elevaría los mismos a la Comisión Nacional. Sin embargo, el mecanismo no funcionó de modo tan aceitado. Muchas sub comisiones locales retrasaron sus envíos y la Comisión Provincial, apurada por las circulares emitidas desde Nación, envió en varias ocasiones la documentación de modo parcial. En el caso de la Provincia de Buenos Aires, la comisión incluso consideró que sus expedientes no debían salir del fuero provincial, distribuyéndose directamente a los jueces del crimen, cuando se acreditaba la existencia de hechos delictuosos, o a los ministerios, municipalidades y otros organismos, cuando se trataba de irregularidades administrativas. ${ }^{18}$ Más radicales en su federalismo fueron respecto a la incautación de bienes. Los integrantes de la Comisión Investigadora Provincial solicitaron la creación de un organismo similar a la Junta de Recuperación Patrimonial para la Provincia de Buenos Aires o, en su defecto y como garantía mínima, instaron a que un organismo del fuero provincial representara sus intereses ante el organismo nacional. Incluso, en un primer momento, la Comisión Provincial

\footnotetext{
18 Nota de Julio Aramburu (presidente comisión PBA) a Mac Lean (presidente comisión nacional), 24/4/56. Archivo Intermedio-Archivo General de la Nación. Fiscalía Nacional de Recuperación Patrimonial. Clasificación y Custodia de Actuaciones. Comisión Provincial (en adelante AGNFNRP.CCA.CP) Buenos Aires. La propia presencia en el archivo de la Fiscalía Nacional de Recuperación Patrimonial de siete cajas correspondientes a la Provincia de Buenos Aires muestran que una buena parte del material fue desconcentrado según indicaba el procedimiento desde nación, aunque desconocemos el volumen del faltante.
} 


\section{Silvana Gabriela Ferreyra}

tomó medidas más directas al negarse a enviar la lista de interdictos al organismo nacional, aduciendo que los investigados no se habían enriquecido con el patrimonio de la nación sino que habían malversado bienes del fisco de la provincia o de las comunas.

Si seguimos pensando desde una perspectiva escalable también podemos partir del contraste de las comisiones nacionales y provinciales para interrogarnos sobre las diferencias entre los procesos de "desperonización". En esta lógica, Fernando Castillo caracterizó el proceso en Jujuy como desigual y gradual, ${ }^{19}$ en contraste con las acciones más decididas y rápidas de la comisión nacional. Por el contrario, aunque bajo el mismo enfoque, para Martha Ruffini en Río Negro se desencadenó una fiebre de denuncias y delaciones explicables a partir del fuerte espíritu revanchista ocasionado por la marginalidad política a la que fue sometida esta región durante la etapa territoriana. ${ }^{20}$ Ambas reflexiones nos invitan a pensar la "desperonización" en niveles, en una clave similar a la que ha diseñado Estela Spinelli para estudiar la diversidad existente entre los partidos políticos. ${ }^{21} \mathrm{El}$ resultado: un mosaico de casos provinciales y locales que devolvería una imagen más compleja de la persecución al peronismo, enriqueciendo las figuraciones que nos ha legado la "leyenda negra". ${ }^{22}$ Junto con la indagación cualitativa, una comparativa entre los sumarios realizados y los enviados a la justicia del crimen en las distintas provincias podría devolver una respuesta particular a la pregunta sobre la radicalidad de la "desperonización". ${ }^{23}$

Sin embargo, este interrogante no sólo puede responderse desde una lectura regional, que opta por la comparación de institucionales provinciales y nacionales para brindar respuestas. Nos interesa recuperar la pregunta sobre la radicalidad de la "desperonización", y desde esa clave, sobre la intensidad del conflicto peronismoantiperonismo. El cambio propuesto reside en aplicar un enfoque microanalítico, enfatizando la perspectiva "desde abajo hacia arriba" que nos brinda el proceso seguido por las comisiones investigadoras a través de sus expedientes. Como ha señalado Mariana Nazar, en esta fuente se pueden indagar las diferencias que

\footnotetext{
${ }^{19}$ Fernando Castillo. Antiperonismo y resistencia en Jujuy durante la Revolución Libertadora. Tesis doctoral, Facultad de Filosofía y Letras, Universidad Nacional de Tucumán, 2014

20 Martha Ruffini. "La revolución libertadora en el sur argentino. Persecución política y antiperonismo en Rio Negro". E-latina, Revista electrónica de estudios latinoamericanos, Vol. 11, $\mathrm{N}^{\circ}$ 41. Buenos Aires, 2012, pp. 37-58. Disponible en <http://iealc.sociales.uba.ar/publicaciones/elatina>. [Consulta: 25/03/2015].

${ }^{21}$ María Estela Spinelli. Los vencedores..., Op. Cit.

${ }^{22}$ Entre ellas, plagadas de acusaciones sobre corrupción, inmoralidad, ineficiencia, personalismo, podemos recuperar a Perón abusando de menores, ministros corruptos manejando discrecionalmente grandes montos de dinero, funcionarios obsecuentes frente a los líderes, empleados cobrando sueldos del estado pero trabajando para el partido, Eva y su megalomanía.

${ }^{23}$ No manejamos una serie de datos completa sobre este punto. Junto a los datos ya señalados para la Provincia de Buenos Aires, podemos agregar: según el presidente de la Comisión Nacional, Leonardo Mc Lean, para Capital Federal la proporción entre expedientes ingresados y enviados a la justicia fue de 15000 y 300 respectivamente; en el Ministerio de Salud Pública, 1100 y 57; en la provincia de Santa Fe (segunda circunscripción) 999 y 150. Estos datos fueron extraídos de Vicepresidencia de la Nación - Comisión Nacional de Investigaciones, Documentación..., Op. Cit., tomo I. La búsqueda de otras fuentes de información podría brindar una perspectiva más completa.
} 


\section{Junta Consultiva y Comisiones Investigadoras en la Provincia de Buenos Aires}

distintas áreas de una institución tienen respecto a la visión sobre determinado problema, e incluso la opinión, mirada y/o participación de otros actores que no forman parte de dicha institución (otras instituciones del Estado, de la sociedad civil, individuos o familias, etc.). ${ }^{24}$

Esta lógica de lo pequeño no está necesariamente anudada a las historias provinciales o locales, pues puede rastrearse tanto en las denuncias entre empleados del ministerio de salud de la Nación como en los enfrentamientos entre vecinos peronistas y antiperonistas en un pueblo del interior bonaerense. No obstante, el análisis de expedientes provino principalmente de quienes han explorado en historias locales y provinciales, pues formularon cuestionarios más sensibles a los aportes de este corpus. El trabajo de Martha Ruffini al que ya hemos citado es una muestra palpable, pues cruza sus reflexiones sobre semejanzas y diferencias de la política territoriana y patagónica en el concierto nacional, con el propósito de advertir el involucramiento de la sociedad civil y sus organizaciones en la persecución y represión de algunos habitantes. ${ }^{25}$

Siguiendo estos aportes, apostamos entonces a poner en diálogo el problema sobre la "desperonización" con un tópico más amplio: el de las actitudes de la "gente corriente" frente a las dictaduras. De este modo, a la pregunta sobre la mayor o menor radicalidad del conflicto político en escenas cotidianas se suma el interrogante sobre sus causalidades. Asimismo, desprovincializamos un problema como el conflicto peronismo-antiperonismo que, de lo contrario, suele explicarse circularmente, en los términos dicotómicos que lo engendran. En esta línea, Fitzpatrick y Gellately han mostrado que un número muy importante de denuncias en la mayoría de las sociedades autoritarias fueron hechas contra gente ordinaria vecinos, compañeros de trabajo, aldeanos- con quienes los denunciantes tenían un trato cotidiano. En estas tramas, presentes en la Alemania Nazi, la Italia Fascista o la Rusia estalinista, lo político se mezclaba con lo personal de múltiples modos, intensificando los enfrentamientos y evidenciando razones diversas para el apoyo a regímenes opresivos. ${ }^{26}$

Hasta qué niveles llegaron las denuncias en la Argentina posperonista queda plasmado en el relato de un maestro rural de la localidad bonaerense de 9 de julio. En su narración, da cuenta de la incautación de una motocicleta que había llegado a

\footnotetext{
${ }^{24}$ Mariana Nazar. "En torno a la especificidad del archivo como territorio de investigación", ponencia presentada en VI jornadas sobre etnografía y métodos cualitativos, Buenos Aires, 2010.

${ }^{25}$ Martha Ruffini. "La revolución libertadora..., Op. Cit.

${ }^{26}$ Sheila Fitzpatrick y Robert Gellately. "Introduction to the Practices of Denunciation in Modern European History, 1789-1989”. The Journal of Modern History, Vol. 68, Nㅜ 4. Chicago, 1996, pp. 747767, The University of Chicago Press. Disponible en <http://www.jstor.org/stable/2946718>. [Consulta: 15/04/2014]. Aunque esta bibliografía ha sido utilizada en la historiografía local para pensar las denuncias durante el peronismo o para indagar sobre la complicidad civil en la dictadura militar abierta por el golpe de estado de 1976, nos parece oportuno retomarla para indagar la coyuntura 55-58. Para el período peronista véase Flavia Fiorucci. "La denuncia bajo el peronismo. El caso del campo escolar". Latin American Research Review, Vol. 48, №1. Pittsburgh, 2013, pp- 3-23. Para el período de la dictadura cívico-militar puede leerse, entre otros trabajos del autor, el publicado en esta revista. Daniel Lvovich. "Actitudes sociales y dictaduras. Las historiografías Española y Argentina en perspectiva comparada". Páginas, Vol.1 №. 1. Rosario, 2008, pp. 29-49.
} 


\section{Silvana Gabriela Ferreyra}

su poder como un inesperado regalo de Perón, tras su pedido personal al gobernador Aloé para sacar un crédito en el Banco Nación que le facilitase el acceso al vehículo. Aunque se declaraba comprensivo frente a las razones del secuestro del rodado, el maestro solicitaba se le brinde otra solución para trasladarse cotidianamente a su trabajo. ${ }^{27}$

Otros expedientes son particularmente ilustrativos de las tramas que se tejían entre lo político y lo personal. Así, una empleada del Ministerio de Hacienda, Economía y Previsión de la Provincia de Buenos Aires denunciaba al subsecretario de Economía y Director de Turismo y Parques y a otros empleados de menor jerarquía por persecución política. La denunciante reclamaba que se le devolvieran los sueldos que le deberían haber sido pagados durante su suspensión por la supuesta destrucción de una fotografía de Juan Domingo Perón y su esposa Eva María Duarte. Al mismo tiempo, exigía que se investigue y exonere a los acusados y se consideren los daños materiales y personales causados, pues durante muchos años no había sido promovida de su cargo. ${ }^{28}$

En la misma línea, ilustra el testimonio de un empleado muy joven del Banco Provincia, llamado a declarar por la comisión investigadora del municipio de Florencio Varela, frente a la cual relató el modo en que solicitó un aval político al entonces intendente municipal para aspirar un puesto de auxiliar. La traducción de su testimonio que hace la comisión, a la vez que nos habla de los protocolos de defensa ensayados por los peronistas frente a una escena inquisidora, trasluce la complejidad del proceso:

Pasados dos meses aproximadamente y habiéndose afiliado al ex partido peronista -se le había indicado que era imprescindible- se apersonó nuevamente al ex intendente y como respuesta se le devolvió la ficha de solicitud que le fuera entregada por la oficina de personal del banco, haciéndole notar que había sido calificado como opositor. Dice que frente a la situación creada el señor padre se apersonó a la delegada censista, Sra. Serafina Tedesco y al insistir en el pedido formulado, se le aconsejó que se hiciera ver acompañado de algunos amigos de la Unidad Básica y que se acordara de Evita llevándole algún ramito de flores de vez en cuando, como así también, que dejara de frecuentar lugares donde concurrían personas no adictas al regimen, a las que estaba vinculado por razones de trabajo. Aceptado en cierta forma el consejo manifiesta que al final le fue resuelto favorablemente el pedido formulado al considerársele ya peronista. Pasado cierto tiempo y ya empleado del Banco Provincia dejó de concurrir a la Unidad Básica y expone que esto fue observado por la delegada censista quien se apersonó a su señor padre y le manifestó que

${ }^{27}$ AGN- FNRP.CCA.CP. Buenos Aires. Comisión investigadora 9 de julio. Caja 3.
${ }^{28}$ Expediente 107085/57. AGN- FNRP.CCA.CP. Buenos Aires. Caja 3. 
como se le había facilitado la obtención del puesto en el banco, en la misma forma se podía disponer su cesantía. ${ }^{29}$

Otro expediente muestra los cruces entre lo personal y lo político de manera aún más descarnada, así como la forma en que las comisiones intensificaron antes que amortiguar los enfrentamientos. La fuente da cuenta de cómo el dueño de un comercio en Mar del Plata, que había sido investigado a raíz de su participación en la Alianza Libertadora Nacionalista, se presenta ante la justicia para defenderse de la injurias de dos vecinos opositores al peronismo, uno de ellos policía. En efecto ambos vecinos habían confirmado que lo consideraban posible autor de las pintadas a favor del "ex dictador" que habían aparecido en inmediaciones de su domicilio, irrumpiendo en su comercio en búsqueda de posibles evidencias. El comerciante señala:

el nombrado, comenzó por decirle palabras agraviantes para un hijo suyo que había fallecido (...) Cree que ello se debe a que el deponente hace cerca de dos meses a la fecha, salió de testigo por un hecho delictuoso en el que se encontraba involucrado su vecino. Que no obstante esas palabras prosiguió después con otras "que la negra de Carmona, que concurría a su comercio, se la había cogido muchas veces" (...) prosiguió diciéndole en el interior de dicho comercio, que en el mismo se hablaba mal del Gobierno Provisional, a lo que el dicente le respondió que si bien tenía la idea de la Alianza Libertadora Nacionalista, luego de haber estado detenido, nunca estuvo haciendo propaganda contra el actual gobierno, ya que el comercio lo posee para mantener a su esposa e hijos y no para propaganda, ya que si fuera verdad, la Policía le hubiera secuestrado panfletos y otros papeles. ${ }^{30}$

Un primer acercamiento a esta secuencia de relatos, que no constituye sino una simple muestra de los cientos que se apilan en el archivo, nos podría llevar a afirmar que el enfrentamiento entre peronistas y antiperonistas en este contexto, debería explicarse más por razones personales que por razones políticas, lo que amortiguaría la relevancia de la dicotomía. No obstante, en líneas generales, creemos que aun cuando no puedan desconocerse dosis variables de oportunismo en las denuncias, su ejecución misma conllevan un importante y ambiguo proceso de identificación política. Si observamos en profundidad, creemos advertir que el proceso de denuncias a "ras de suelo" que se desata en los meses posteriores al golpe de estado de 1955 nos muestra sujetos que buscan recomponer un orden social que el peronismo había alterado. En concreto, las denuncias parecen dejar entrever los quiebres que la emergencia del peronismo había producido en el orden local. Desde esta perspectiva, lejos de domesticar el impacto de la brecha entre peronismo y

\footnotetext{
${ }^{29}$ Expediente 2006/56. AGN- FNRP.CCA.CP. Buenos Aires. Sub comisión Florencio Varela. Caja 3.

${ }^{30}$ Expediente 2074/1957. Archivo Tribunales. Departamento Judicial Mar del Plata.
} 


\section{Silvana Gabriela Ferreyra}

antiperonismo, una visión local de la "desperonización" nos muestra su faceta más radical.

\section{Conclusión}

Este artículo ha intentado reflexionar en la intersección entre dos tópicos: las historias locales y los estudios sobre antiperonismo en la "libertadora". Aunque el artículo busca extender su campo de reflexión más allá de estos confines, nuestro punto de partida podría vincularse con una historia política tradicional, pues recortamos dos instituciones creadas por el golpe de 1955: juntas consultivas y comisiones investigadoras. Ambas son fácilmente escalables desde una lógica política y administrativa, pues se replicaban a nivel nacional, provincial y local.

Atendiendo a esta cualidad, quizás más evidente, apuntamos a resaltar la especificidad de la "libertadora" en cada espacio, analizando las similitudes y diferencias con los restantes niveles al aplicar la lógica modelo/desviaciones. Por esta vía recuperamos el trabajo de César Tcach, quien en el desarrollo de esta propuesta muestra cómo el seguimiento de estas experiencias para el escenario cordobés devolvía escenas menos impregnadas por el conflicto peronismoantiperonismo que las que los relatos nacionales habían captado. Una parte de nuestro análisis devuelve conclusiones similares. Por un lado, como ya ha mostrado Spinelli, las discrepancias partidarias al seno del antiperonismo eran intensas y variadas. Pero las lecturas provinciales muestran que las alianzas y coaliciones no siempre se daban del mismo modo y que las posiciones radicalizadas y moderadas se reflejaban también en diferencias regionales al seno de cada partido. Asimismo, las variaciones en la política de "desperonización" no sólo se relacionaban con diferencias partidarias sino también con lógicas regionales. Junto con la evidencia de un escenario más complejo y heterogéneo del que conocíamos, aparece la centralidad de otras contradicciones, como federalismo/centralismo y oposición/oficialismo, que tejen tramas políticas alternativas al binomio peronismo/antiperonismo. De este modo, se consolida la tesis sobre la heterogeneidad del antiperonismo ${ }^{31}$ que, si bien no invalida el alineamiento en campos enfrentados, habilita el debate sobre al intensidad del conflicto en cada contexto histórico, al punto de discutirse la pertinencia o no de este clivaje para pensar la política argentina post 55 .

\footnotetext{
${ }^{31}$ Como dos caras de la misma moneda, la literatura revisionista y las visiones patológicas sobre el peronismo comparten una visión bifronte sobre el mundo político. En un esfuerzo por no reforzar estereotipos y visiones dicotómicas de la Argentina, la historiografía académica de las últimas décadas ha construido una visión "normalizadora", donde el peronismo habría aportado a la democratización a partir de la ampliación de la ciudadanía social, aunque este proceso habría ido en detrimento del avance del pluralismo y los derechos civiles. En este marco, los trabajos sobre antiperonismo han buscado aportar heterogeneidad a un campo que se visualizaba como homogéneo. Para un análisis de la normalización del peronismo véase Omar Acha y Nicolás Quiroga. El hecho maldito. Conversaciones para otra historia del peronismo. Rosario, Prohistoria, 2012.
} 


\section{Junta Consultiva y Comisiones Investigadoras en la Provincia de Buenos Aires}

Otra perspectiva devuelve el estudio de las comisiones investigadoras. En efecto, resulta difícil devolver imágenes que diluyan el peso del conflicto peronismoantiperonismo al analizar esta institución, pues el propio objetivo de "desperonizar" devolvía -ya desde su significación- el binomio al centro de la escena. Aun así, la lógica modelo/desviaciones y una mirada cupular logran desdibujar las aristas del enfrentamiento: algunos escenarios provinciales mostraban lógicas menos excluyentes y la cuestión federal tomaba centralidad en algunos planteos. Asimismo, una mirada "desde arriba" puede analizar la dinámica de la "desperonización" a partir de cálculos electorales o chances de gobernabilidad mostrando al conflicto peronismo-antiperonismo prácticamente como "fachada" para conseguir adhesiones. Por el contrario, una mirada microanalítica, que atiende a la recepción de las políticas "desperonizadoras" entre la "gente ordinaria" difumina esas diferencias y resalta la intensidad del conflicto. Los cruces entre lo personal y lo político que evidencian las denuncias muestran la tensión social que una década de peronismo había instalado a "ras de suelo". Así, figuraciones que referían a la desperonización en términos de combate a la corrupción, personalismo e ineficiencia del régimen se proyectaban a nivel local en un plano más profundo, como un ataque a las transformaciones políticas, sociales y culturales acontecidas en la década peronista.

En resumen, es posible que todas estas reflexiones no alcancen para considerar que algunas de las hipótesis sean más veraces que otras. La intensidad del conflicto peronismo-antiperonismo parece moderarse en los ámbitos dirigenciales y radicalizarse en las expresiones políticas de la gente ordinaria. Las historias locales, como caso o como dimensión, brindan posibilidades para hurgar en ambos escenarios. Bernard Lepetit sugiere que sí existen escalas más pertinentes que otras para desarrollar ciertas problemáticas y testear ciertas hipótesis, es preciso establecer tan sistemáticamente cuanto sea posible las consecuencias provocadas por las variaciones de escala del campo recortado. En definitiva, considera que la cuestión de saber en qué campo de validación y explicación se puede situar en función de la escala de observación escogida, tal vez no tenga otra solución que la práctica. ${ }^{32}$ En esa línea, este artículo puede considerarse un pequeño laboratorio de trabajo.

\section{Bibliografía}

Omar Acha y Nicolás Quiroga. El hecho maldito. Conversaciones para otra historia del peronismo. Rosario, Prohistoria, 2012.

Osvaldo Barrenche (director). Historia de la Provincia de Buenos Aires. Tomo 5: Del primer peronismo a la crisis de 2001. Buenos Aires, UNIPE- Edhasa, 2014.

\footnotetext{
32 Bernard Lepetit. "Sobre a escala na história", en Jacques Revel, (editor), Jogos de escala: a experiencia da micro-analis. Rio de Janeiro, Fundación Getulio Vargas, 1998.
} 


\section{Silvana Gabriela Ferreyra}

Aixa Bona y Juan Vilaboa. "La transición política de los territorios nacionales y la caída del peronismo: el caso de Santa Cruz”. Estudios, № 22. Córdoba, 2009, pp. 4960.

Fernando Castillo. Antiperonismo y resistencia en Jujuy durante la Revolución Libertadora. Tesis doctoral, Facultad de Filosofía y Letras, Universidad Nacional de Tucumán, 2014.

Luciano De Privitellio. Vecinos y ciudadanos. Buenos Aires, Siglo XXI, 2003.

Marcela Ferrari y Mariana Pozzoni. "Tensiones y conflictos en el peronismo: un análisis a través de la Legislatura bonaerense, 1973-1976“. Cahiers des Amériques latines, $\mathrm{N}^{\circ}$ 75. París, 2014, pp. 147-176.

Silvana Ferreyra. Socialismo y antiperonismo: el Partido Socialista Democrático. Transformación partidaria y dinámica política en tiempos de proscripción (Provincia de Buenos Aires, 1955-1966). Tesis de Doctorado. Mar del Plata, UNMdP, 2012.

Flavia Fiorucci. "La denuncia bajo el peronismo. El caso del campo escolar". Latin American Research Review, Vol. 48, №1. Pittsburgh, 2013, pp-3-23.

Sheila Fitzpatrick y Robert Gellately. "Introduction to the Practices of Denunciation in Modern European History, 1789-1989". The Journal of Modern History, Vol. 68, ${ }^{\circ}$ 4. Chicago, 1996, pp. 747-767, The University of Chicago Press. Disponible en <http://www.jstor.org/stable/2946718>. [Consulta: 15/04/2014].

Graciela Iuorno. "La provincialización de Río Negro. Interregno y conflictos de intereses nacionales y locales", en Graciela Iuorno y Edda Crespo, (coordinadoras), Nuevos Espacios. Nuevos problemas. Los territorios nacionales. Neuquén, EducoUniversidad Nacional del Comahue-Universidad Nacional de la Patagonia San Juan Bosco-Cehepyc Editores, 2008.

Bernard Lepetit. "Sobre a escala na história”, en Jacques Revel, (editor), Jogos de escala: a experiencia da micro-analis. Rio de Janeiro, Fundación Getulio Vargas, 1998. Ricardo Levene. Historia de la Provincia de Buenos Aires y formación de sus pueblos. La Plata, Taller de Impresiones Oficiales, 1941.

Leandro Lichtmajer y Lucía Santos Lepera. "Repensando el golpe de 1955 desde una perspectiva provincial: Iglesia católica y radicalismo en Tucumán", ponencia presentada en Tercer Congreso de Estudios sobre el Peronismo, San Salvador de Jujuy, Jujuy, 2012.

Daniel Lvovich. "Actitudes sociales y dictaduras. Las historiografías Española y Argentina en perspectiva comparada". Páginas, Vol.1 №. 1. Rosario, 2008, pp. 29-49. Darío Macor y César Tcach, (editores). La invención del peronismo en el interior del país. Santa Fe, Ediciones UNL, 2003.

Darío Macor y César Tcach, (editores.). La invención del peronismo en el interior del país II. Santa Fe, Ediciones UNL, 2013.

Mariana Nazar. "En torno a la especificidad del archivo como territorio de investigación", ponencia presentada en VI jornadas sobre etnografía y métodos cualitativos, Buenos Aires, 2010. 


\section{Junta Consultiva y Comisiones Investigadoras en la Provincia de Buenos Aires}

Elías Palti. “De la historia de 'ideas' a la historia de los 'lenguajes políticos'. Las escuelas recientes de análisis conceptual. El panorama latinoamericano". Anales Nueva Época, № 7/8. 2004, pp. 63-81.

Jacques Revel. "Microanálisis y construcción de lo social". Anuario del IEHS, № 10. Tandil, 1995, pp. 125-143.

Alain Rouquié. Poder militar y sociedad política en la Argentina,1943-1973, Vol. II. Buenos Aires, Emecé.

Martha Ruffini. "La revolución libertadora en el sur argentino. Persecución política y antiperonismo en Rio Negro". E-latina, Revista electrónica de estudios latinoamericanos, Vol. 11, $\mathrm{N}^{\circ}$ 41. Buenos Aires, 2012, pp. 37-58. Disponible en $<$ http://iealc.sociales.uba.ar/publicaciones/e-latina >. [Consulta: 25/03/2015].

Justo Serna y Anaclet Pons. "Más cerca, más denso. La historia local y sus metáforas", en Sandra Fernández, (compiladora), Más allá del territorio. La historia regional y local como problema. Discusiones, balances y proyecciones. Rosario, Prohistoria, 2007.

María Estela Spinelli. Los vencedores vencidos. El antiperonismo y la "revolución libertadora." Buenos Aires, Biblos, 2005 y por María Sáenz Quesada. La Libertadora. De Perón a Frondizi. 1955-1958. Historia pública y secreta. Buenos Aires, Sudamericana, 2007.

César Tcach. "Partidos y pactos políticos en la Córdoba Libertadora (1955-1958)". Revista Estudios, № 3. Córdoba, 1994, pp. 17-30.

Marcela Ternavasio. Municipio y política, un vínculo conflictivo. Tesis de Maestría. Buenos Aires, FLACSO, 1991.

Recibido: $14 / 03 / 2016$

Evaluado: $10 / 04 / 2016$

Versión Final: 25/04/2016 